\title{
Parental understanding of wheeze and its impact on asthma prevalence estimates
}

\section{To the Editors:}

In an article recently published in the European Respiratory Journal, Michel et al. [1] presented the results of a study assessing the accuracy of the response to a question on wheezing in the last 12 months, by comparing responses with parental understanding on wheezing, and building three different scenarios (A, B and C). In the study, Michel et al. [1] accurately raised methodological limitations on estimates of asthma prevalence rates based on such a question and, hence, questionnaires. Although we subscribe to the limitations raised in the paper, we would like to make comments on three statements posed by the authors.

First, the authors stated that "questionnaire surveys are, therefore, more likely to underestimate the true prevalence of wheeze, not the converse". Indeed, all the prevalence rates observed by the authors in the total study population and in subgroups were lower than the rates estimated in scenario $B$, in which children whose parents had not reported wheeze and given an incorrect definition of wheeze were considered as having asthma. However, this scenario seems to be an extreme situation. If observed rates are compared with scenario C, which the authors describe as "realistic" and maybe closer to the true rate, in only two subgroups (South Asian and the most deprived) would the observed prevalence rates underestimate the true values. It is worth noting that the estimated rates in the three different scenarios are a function of the observed rates. For instance, if the observed prevalence was $20 \%$ in both these subgroups and the proportion of correct description among those who reported wheeze and among those who did not report wheeze were as in the study by Michel et al. [1], the estimates in scenario $C$ would be very similar to the observed rates of $20 \%$; that is, there would not be underestimation (table 1). Estimates of $\geqslant 20 \%$ have been observed in International Study of Asthma and Allergies in Childhood (ISAAC) surveys in several settings in Latin America and in English-speaking countries [2,3]. In conclusion, it seems to us

\begin{tabular}{|c|c|c|c|c|}
\hline $\begin{array}{r}\text { TABLE } 1 \\
\text { P } \\
\text { S } \\
\text { O }\end{array}$ & \multicolumn{4}{|c|}{$\begin{array}{l}\text { Prevalence rates of asthma according to } \\
\text { subgroups and different scenarios, based on } \\
\text { observed rates and a simulated rate of } 20 \%\end{array}$} \\
\hline \multirow[t]{2}{*}{ Subgroups } & \multirow{2}{*}{$\begin{array}{c}\text { Observed } \\
\text { prevalence \% }\end{array}$} & \multicolumn{3}{|c|}{ Scenarios \% } \\
\hline & & A & B & C \\
\hline \multicolumn{5}{|l|}{ South Asian } \\
\hline Michel et al. & 13.5 & 10.0 & 38.3 & 14.2 \\
\hline Simulation & 20.0 & 14.8 & 41.0 & 20.5 \\
\hline \multicolumn{5}{|l|}{ Most deprived } \\
\hline Michel et al. [1] & 15.8 & 12.3 & 35.1 & 16.1 \\
\hline Simulation & 20.0 & 15.6 & 37.2 & 20.1 \\
\hline
\end{tabular}

that the statement saying there would be an underestimation of the true prevalence observed in surveys can not be taken as a rule.

Secondly, Michel et al. [1] suggest that results from the main analyses using a general definition for asthma "should be confirmed by a secondary analysis including only those with more severe wheezing", since rates of severe asthma are more reliable. Although the authors specify this as a secondary analysis, we would like to remind readers of the fact that risk factors related to the frequency of disease are not the same as risk factors related to severity (prognosis). Even if the proportions of total asthma were different in distinct groups, for example according to socioeconomic status (SES), the proportions of severe asthma may be the same in each group. This could happen, for instance, if the SES group with the higher prevalence of total asthma had a lower proportion of prognostic factors, thus lowering the rates of severe asthma towards those of the other SES group. The similarity in prevalence between the two SES groups based on severe cases would, therefore, be real, but would not imply that the differences in prevalence of total asthma were invalid. Therefore, findings from the main analyses regarding total asthma should not necessarily be cast in doubt if results from secondary analyses using only severe cases are inconsistent.

Thirdly, the authors suggest that the presence of atopy should be used to validate the diagnosis of asthma. Indeed, atopy is strongly associated with asthma and, thus, asthma cases with atopy would have a more accurate definition. However, asthma is a syndrome with different aetiologies [4] and defining asthma according to atopy selects a specific phenotype, atopic asthma. Instead of using atopy to increase the validity of the diagnosis, prevalence rates in different subpopulations should be shown separately for atopic and nonatopic asthma.

\section{S.S. Cunha* and P.J. Newcombe ${ }^{\#}$}

*Instituto de Saúde Coletiva, Universidade Federal da Bahia, Bahia, Brazil. "London School of Hygiene and Tropical Medicine, London, UK.

\section{STATEMENT OF INTEREST}

None declared.

\section{REFERENCES}

1 Michel G, Silverman M, Strippoli MP, et al. Parental understanding of wheeze and its impact on asthma prevalence estimates. Eur Respir J 2006; 28: 1124-1130.

2 Asher MI, Montefort S, Bjorksten B, et al. Worldwide time trends in the prevalence of symptoms of asthma, allergic rhinoconjunctivitis, and eczema in childhood: ISAAC Phases 
One and Three repeat multicountry cross-sectional surveys. Lancet 2006; 368: 733-743.

3 Mallol J, Sole D, Asher I, Clayton T, Stein R, Soto Quiroz M. Prevalence of asthma symptoms in Latin America: the International Study of Asthma and Allergies in Childhood (ISAAC). Pediatr Pulmonol 2000; 30: 439-444.

4 A plea to abandon asthma as a disease concept. Lancet 2006; 368: 705 .

DOI: $10.1183 / 09031936.00004207$

From the authors:

We would like to thank S.S. Cunha and P.J. Newcombe for their discussion of our paper. Although we generally agree with most arguments raised by the two authors, we want to emphasise that their points did not accurately represent our carefully worded statements.

First, using different scenarios, we have shown how estimates of the prevalence of wheeze differ if understanding of the word "wheeze" by parents is taken into account [1]. In our paper, table 4 shows that misunderstanding of "wheeze" does not, as often postulated, invariably lead to an overestimate of the prevalence of wheeze, but might also lead to an underestimate as shown for children of south Asian backgrounds and deprived neighbourhoods. We did not claim that "underestimation can be taken as a rule". The fact that we presented different scenarios, with some showing higher estimates than the observed results and others lower, confirms this. We thank the authors for demonstrating that estimates also depend on baseline prevalence.

Secondly, we agree that risk factors for prevalence are not necessarily risk factors for severity (although they often are). However, we do not agree that severe childhood wheeze can be equated with a poor prognosis and, therefore, that risk factors for severity are the same as risk factors for prognosis. Preschool children with viral-induced wheeze can be very severely ill and hospitalised but still have a good long-term prognosis, as they are likely to grow out of these problems. When we propose to confirm, in a population-based study, important results by a sensitivity analysis "including only those with more severe wheezing" (such as those with more frequent attacks or with disturbed sleep), we think that such a definition would in fact merely exclude those children whose parents have noticed a respiratory noise at some time, and a large proportion of such "more severe" cases would not have even been treated with a bronchodilator [2].

Thirdly, regarding atopy, we have not stated that "presence of atopy is necessary to validate wheeze". Our wording was to "use objective measurements (such as bronchial responsiveness and allergy tests) to validate findings in subgroups of the population". We have been well aware of the existence of different phenotypes within the spectrum of wheezing disorders in childhood for many years [3-5]. We entirely agree that the presence of atopy is not necessary to diagnose, for instance viral-induced wheeze in young children, but this was not the topic of our paper.

\section{C.E. Kuehni*, G. Michel*, M-P.F. Strippoli*, M. Zwahlen*, A.M. Brooke", J. Grigg ${ }^{\#}$ and M. Silverman ${ }^{\#}$}

*Swiss Paediatric Respiratory Research Group, Dept of Social and Preventive Medicine, University of Berne, Berne, Switzerland. "The Leicester Children's Asthma Centre, Division of Child Health, Dept of Infection, Immunity \& Inflammation, University of Leicester, Leicester, UK.

\section{STATEMENT OF INTEREST}

None declared.

\section{REFERENCES}

1 Michel G, Silverman M, Strippoli MP, et al. Parental understanding of wheeze and its impact on asthma prevalence estimates. Eur Respir J 2006; 28: 1124-1130.

2 Chauliac ES, Silverman M, Zwahlen M, Strippoli MP, Brooke AM, Kuehni CE. The therapy of pre-school wheeze: appropriate and fair? Pediatr Pulmonol 2006; 41: 829-838.

3 Silverman M. Out of the mouths of babes and sucklings: lessons from early childhood asthma. Thorax 1993; 48: 1200-1204.

4 Childhood Asthma and other Wheezing Disorders. 1st Edn. Silverman M, ed. London, Chapman \& Hall, 1995.

5 Kuehni CE, Davis A, Brooke AM, Silverman M. Are all wheezing disorders in very young (preschool) children increasing in prevalence? Lancet 2001; 357: 1821-1825.

DOI: 10.1183/09031936.00009507 